\title{
Development of a High-Sensitivity Quantitation Method for Arginine Vasopressin by High-Performance Liquid Chromatography Tandem Mass Spectrometry, and Comparison with Quantitative Values by Radioimmunoassay
}

\author{
Yasuko TsukaZaki, ${ }^{1 \dagger}$ Naoto Senda, ${ }^{* 1, * 2}$ Kinya Kubo, ${ }^{* 2}$ Shigeru Yamada, ${ }^{* 3}$ Hiroyuki KugOH, $* 2, * 4$ \\ Yasuhiro KAZUKI, ${ }^{* 2, * 4}$ and Mitsuo OSHIMURA $* 2$ \\ *1 Analytical Technology Research Department, LSI Medience Corp., 2-1-6 Sengen, Tsukuba, \\ Ibaraki 305-0047, Japan \\ *2 Chromosome Engineering Research Center, Tottori University, 86 Nishi-cho, Yonago, Tottori 683-8503, Japan \\ *3 K. K. AB SCIEX, 4-7-35 Kitashinagawa, Shinagawa, Tokyo 140-0001, Japan \\ *4 Department of Biomedical Science, Institute of Regenerative Medicine and Biofunction, Graduate School of \\ Medical Science, Tottori University, 86 Nishi-cho, Yonago, Tottori 683-8503, Japan
}

\begin{abstract}
Human plasma arginine vasopressin (AVP) levels serve as a clinically relevant marker of diabetes and related syndromes. We developed a highly sensitive method for measuring human plasma AVP using high-performance liquid chromatography tandem mass spectrometry. AVP was extracted from human plasma using a weak-cation solid-phase extraction plate, and separated on a wide-bore octadecyl reverse-phase column. AVP was quantified in ion-transition experiments utilizing a product ion $(\mathrm{m} / \mathrm{z}, 328.3)$ derived from its parent ion $(\mathrm{m} / \mathrm{z}, 542.8)$. The sensitivity was enhanced using $0.02 \%$ dichloromethane as a mobile-phase additive. The lower limit of quantitation was $0.200 \mathrm{pmol} / \mathrm{L}$. The extraction recovery ranged from $70.2 \pm 7.2$ to $73.3 \pm 6.2 \%$ (mean $\pm \mathrm{SD}$ ), and the matrix effect ranged from $1.1-1.9 \%$. Quality-testing samples revealed interday/intraday accuracy and precision ranging over $0.9-3 \%$ and $-0.3-2 \%$, respectively, which included the endogenous baseline. Our results correlated well with radioimmunoassay results using 22 human volunteer plasma samples.
\end{abstract}

Keywords Arginine vasopressin, plasma, mass spectrometry, liquid chromatography, matrix effect, analytical sensitivity, analytical specificity, electrode, accuracy, radioimmunoassay

(Received May 11, 2015; Accepted August 12, 2015; Published February 10, 2016)

\section{Introduction}

Arginine $\left[\mathrm{Arg}^{8}\right]$-vasopressin (AVP) is a neurohypophyseal hormone that is synthesized in hypothalamic cells. The amino acid sequence of AVP is Cys-Tyr-Phe-Gln-Asn-Cys-Pro-ArgGly- $\mathrm{NH}_{2}$, which has a monoisotopic mass of 1083.44 and a disulfide bond formed between Cys1 and Cys6. AVP increases the urine osmolality and the concentrations of urinary components; thus, this hormone pays a critical role in the regulation of water retention. ${ }^{1,2}$ The most common type of diabetes insipidus in humans is the neurological form, central diabetes insipidus, which involves a deficiency of AVP. The second-most common type of diabetes insipidus is nephrogenic diabetes insipidus, which is due to kidney or nephron dysfunction caused by an insensitivity of the kidneys or nephrons to AVP.3,4 Furthermore, AVP causes the vasoconstriction of peripheral blood vessels, which leads to increased arterial pressure. ${ }^{5,6}$ In contrast, the syndrome of inappropriate secretion of antidiuretic hormone (SIADH) is caused by over-secretion of AVP. As a

$\dagger$ To whom correspondence should be addressed.

E-mail: yasukotsukazaki1416560@gmail.com result, an excess of plasma sodium can lead to water retention. ${ }^{7}$ Therefore, precise quantitation of AVP in human plasma is important for correct clinical diagnosis. Previous methods for the quantitation of AVP in human plasma have utilized a radioimmunoassay (RIA) approach. ${ }^{8-11}$ Although these methods are known for their high sensitivity, problems with specificity have been reported. ${ }^{12}$

Liquid chromatography (LC) mass spectrometry (MS) is a high-performance and generally applicable technique used for the quantitation of many drugs, peptides, and proteins in biological samples. In particular, LC tandem MS (LC-MS/MS) is highly sensitive and specific, and does not require the manufacturing of specialized immune reagents, as is the case for RIA and enzyme-linked immunosorbent assays. Peptides and proteins are widely quantified by LC-MS/MS, and MS is an increasingly important analytical technology from the standpoint of clinical laboratories. ${ }^{13}$ The quantitation of AVP using capillary LC-MS/MS has been reported previously. ${ }^{14,15}$ However, the results from these studies did not demonstrate that the LC-MS/MS method had more sensitivity than a standard RIA. In 2014, Zhang et al. ${ }^{16}$ reported a highly sensitive LC-MS/MS assay developed for the quantitation of AVP in human plasma and urine with a lower limit of quantitation (LLOQ) of $1 \mathrm{pg} / \mathrm{mL}$, 
which translates to $0.923 \mathrm{pmol} / \mathrm{L}$. There is a correlation between the plasma osmolality and the AVP level in human plasma. When the osmolality is $260-290 \mathrm{mOsmol} / \mathrm{kg}$, urine excretion is normal and the AVP concentration in plasma is less than $1 \mathrm{pg} / \mathrm{mL} .{ }^{17}$ Furthermore, partial neurogenic diabetes insipidus is associated with a very low AVP plasma concentration because of increased sensitivity to the antidiuretic effect of AVP. In contrast, partial nephrogenic diabetes insipidus is characterized by decreased sensitivity to the antidiuretic effects of normal plasma AVP concentrations. ${ }^{18}$ Therefore, based on our understanding of the necessity for protein measurements in biological samples, we obtained a patent for this protein quantitation method, which uses mass spectrometry. ${ }^{19}$ Furthermore, we designed the current study to develop and validate a more highly sensitive and selective LC-MS/MS method for AVP quantification in human plasma and demonstrate its applicability.

\section{Experimental}

\section{Chemicals and reagents}

All chemical reagents used for sample pretreatment and chromatography were of analytical grade. AVP was purchased from Peptide Institute, Inc. (Osaka, Japan). Desmopressin (DDAVP) was purchased from Sigma-Aldrich (St. Louis, MO). $\mathrm{NH}_{2}$-CY-(F)*-QNCP-(D-Arg)-G-CONH ${ }_{2} ; \quad$ (F)* ${ }^{*}$ Phe + 10; disulfide bridge: Cys1-Cys6 (monoisotopic mass, 1093.44) was used as an internal standard (IS) for AVP, and was purchased from Bio Synthesis, Inc. (Lewisville, TX). Methanol, acetonitrile, phosphoric acid, formic acid, acetic acid, and dichloromethane were purchased from Wako Pure Chemicals Industries, Ltd. (Osaka, Japan). Ultrapure water was prepared using a Milli-Q Synthesis system from Merck Millipore (Billerica, MA). Control serum for calibration was purchased from Nissui Pharmaceutical Co., Ltd. (Tokyo, Japan). Human pooled plasma for quality control (QC) samples was purchased from Bio-Rad Laboratories Inc. (Hercules, CA). A $2 \times 10^{-7}$ $\mathrm{mol} / \mathrm{L}$ polypropylene glycol (PPG) solution was purchased from AB SCIEX (Framingham, MA).

The main stock and working solutions were prepared with $1 \%$ acetic acid in 5\% acetonitrile. The concentration of the stock solution of AVP was $0.5 \mathrm{mmol} / \mathrm{L}$. The concentration of the IS stock solution was $1 \mathrm{mmol} / \mathrm{L}$, and this solution was stored at $-80^{\circ} \mathrm{C}$ in $1.5 \mathrm{~mL}$ siliconized polypropylene tubes (WHATOSON Co., Ltd.; Tokyo, Japan). All dilution series of the standard AVP solution (2.00 to $800 \mathrm{pmol} / \mathrm{L}, 7$ points) were prepared by dilution with $1 \%$ acetic acid in $5 \%$ acetonitrile. A $20 \mathrm{pmol} / \mathrm{L}$ solution of DDAVP was added to the calibration standard solution of AVP to demonstrate that this peptide did not interfere with AVP. Standard solutions for QC samples were prepared at concentrations of $8.00,80.0$, and $1280 \mathrm{pmol} / \mathrm{L}$ for AVP. The IS standard solution was prepared at a concentration of $1200 \mathrm{pmol} / \mathrm{L}$. The stock and standard solutions were stored at $-80^{\circ} \mathrm{C}$ in siliconized polypropylene tubes.

\section{Materials}

An Oasis ${ }^{\circledR}$ WCX $\mu$ Elution plate for solid-phase extraction (SPE) was purchased from Waters Corp. (Milford, MA). A narrow electrode (diameter: $65 \mu \mathrm{m}$ ) was purchased from $\mathrm{AB}$ SCIEX (Framingham, MA).

\section{Serum and plasma samples}

Control serum (AVP-free) used for instrument calibration was purchased from Nissui Pharmaceutical Co., Ltd. (Tokyo, Japan).
Human pooled plasma (with ethylenediaminetetraacetic acid) for QC samples was purchased from Bio-Rad Laboratories Inc. (Hercules, CA). Twenty-two de-identified human plasma samples from volunteers were provided from the Nagoya University Graduate School of Medicine (Nagoya, Japan) and the associated hospitals. These plasma samples were stored at $-80^{\circ} \mathrm{C}$ until analysis. In addition, we performed measurements after receiving approval from the Ethics Committee of the Nagoya University Graduate School of Medicine.

\section{Preparation of standards and QC samples}

A $100-\mu \mathrm{L}$ sample of control serum was used as a surrogate matrix to prepare calibration curves for determining human plasma concentrations. The nominal AVP concentrations of calibration samples were $0.200,0.400,2.00,4.00,20.0,40.0$, and $80.0 \mathrm{pmol} / \mathrm{L}$. The same procedure was also used for the creation of QC samples at three concentration levels, denoted as low QC (endogenous baseline $+0.400 \mathrm{pmol} / \mathrm{L}$ AVP), middle QC (endogenous baseline $+4.00 \mathrm{pmol} / \mathrm{L}$ AVP), and high QC (endogenous baseline $+64.0 \mathrm{pmol} / \mathrm{L}$ AVP). This covers the full range of expected AVP levels that can be found in human pooled plasma. The endogenous baseline of AVP was confirmed with pooled human plasma that was not spiked with AVP.

\section{Sample preparation}

The SPE method for AVP was slightly modified from the method used for quantitating oxytocin in human plasma. ${ }^{20}$ A $100-\mu \mathrm{L}$ sample of a human plasma sample, $10 \mu \mathrm{L}$ of a $1 \%$ acetic acid aqueous solution containing 5\% acetonitrile, and $5 \mu \mathrm{L}$ of an IS solution were mixed. Then, the mixture was diluted with $100 \mu \mathrm{L}$ of a $4 \%$ phosphoric acid aqueous solution. The above mentioned samples were applied to an Oasis ${ }^{\circledR}$ WCX $\mu$ Elution plate after it being conditioned sequentially with $200 \mu \mathrm{L}$ of acetonitrile and $200 \mu \mathrm{L}$ of ultrapure water. After loading, the plate was washed with $200 \mu \mathrm{L}$ of a $5 \%$ ammonia aqueous solution and $200 \mu \mathrm{L}$ of a $75 \%$ acetonitrile aqueous solution, and eluted with $100 \mu \mathrm{L}$ of a $75 \%$ acetonitrile aqueous solution containing $1 \%$ formic acid. The eluate was dried at $50^{\circ} \mathrm{C}$ under a nitrogen stream for $20 \mathrm{~min}$. These residues were reconstituted with $50 \mu \mathrm{L}$ of a $1 \%$ acetic acid aqueous solution containing $5 \%$ acetonitrile.

\section{LC conditions}

High-performance LC separation was performed with a Prominence LC-30A pump coupled with an SIL-30ACMP Autosampler and a Prominence CTO-20A column oven (Shimadzu Co.; Kyoto, Japan), using an XBridge ${ }^{\mathrm{TM}}$ BEH300 C18 $(2.1 \times 50 \mathrm{~mm}, 3.5 \mu \mathrm{m})$ reverse-phase column (Waters Co.; Milford, MA). For each sample, $35 \mu \mathrm{L}$ was injected. A binary gradient was used: mobile phase A consisted of ultra-purified water, $0.01 \%$ of acetic acid; mobile phase B consisted of methanol, $0.02 \%$ dichloromethane. The gradient program was as follows: mobile phase A was held at $100 \%$ for $1.5 \mathrm{~min}$, then decreased linearly to $20 \%$ within $4 \mathrm{~min}$. The divert valve was switched from waste to ion source at $3.5 \mathrm{~min}$. The flow rate was $0.15 \mathrm{~mL} / \mathrm{min}$ for between 0 and $5.5 \mathrm{~min}$. After switching the divert valve from ion source to waste, the system was washed using a flow rate of $0.6 \mathrm{~mL} / \mathrm{min}$, mobile phase B was held at $100 \%$ from 5.51 to $6.0 \mathrm{~min}$, but then decreased linearly to $20 \%$ mobile phase A within $0.5 \mathrm{~min}$. This was followed by an increase to $100 \%$ mobile phase A within $0.1 \mathrm{~min}$, and then holding for $0.4 \mathrm{~min}$. Then, the linearity in mobile phase A was decreased to $0 \%$ within $0.5 \mathrm{~min}$, and held for $2 \mathrm{~min}$. After the washout process, the system was equilibrated for $1.5 \mathrm{~min}$ before the next injection, for a total run time $11 \mathrm{~min}$. 

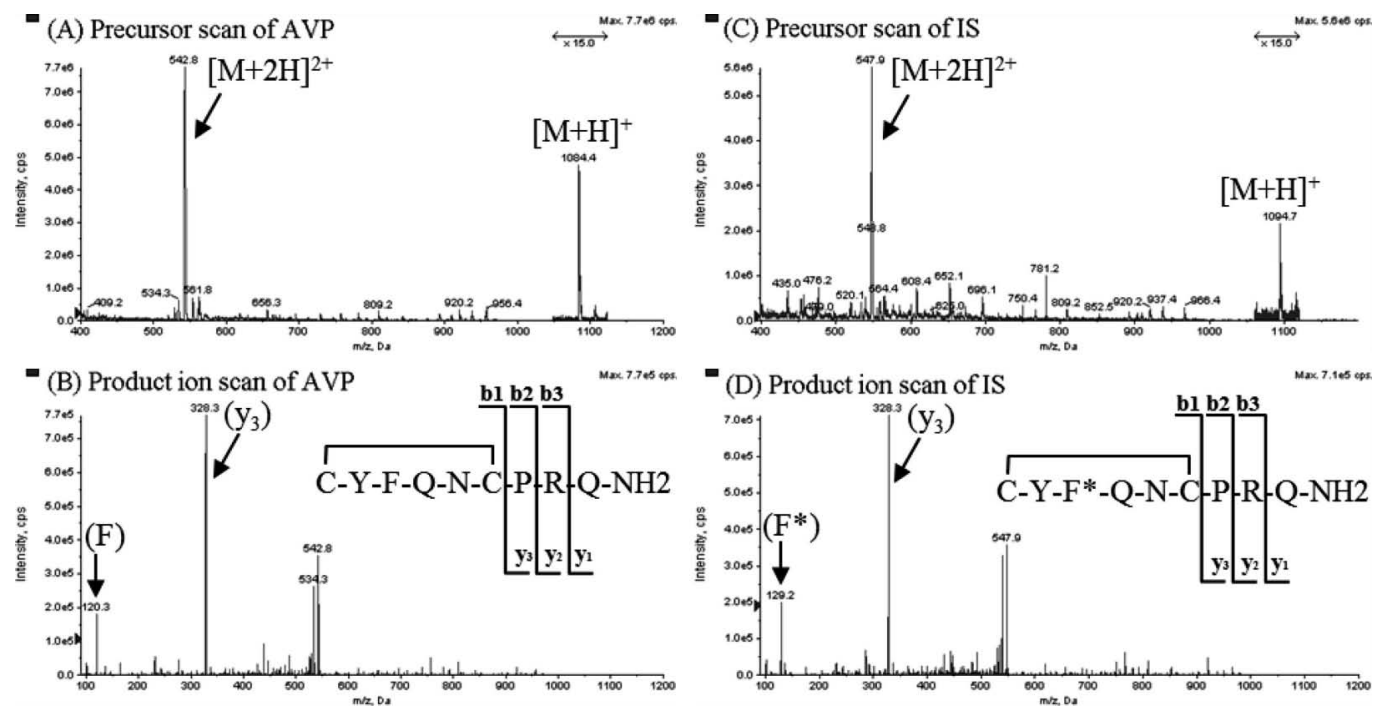

Fig. 1 ESI-positive mass spectra of AVP and IS. Precursor ion mass spectra of AVP (A) and stably labeled AVP (IS) (C). Product ion mass spectra of AVP (B) and stably labeled AVP (IS) (D). Both compounds have a disulfide link between the two cysteine residues, and thus y-type fragmentation occurs, yielding $\mathrm{y}_{3}$ ions as the predominant products.

\section{Mass spectrometry conditions}

The LC-MS/MS method development for the quantitation of AVP in human plasma was performed with a QTRAP ${ }^{\circledR 5500}$ hybrid triple quadrupole-linear ion trap mass spectrometer (AB SCIEX; Framingham, MA), equipped with a Turbo V ion source operated in the electrospray ionization (ESI) positive mode. In addition, the sensitivity of the mass spectrometer was adjusted using a $2 \times 10^{-7} \mathrm{~mol} / \mathrm{L}$ PPG solution as follows. The voltage of the detector (CEM) and focusing lens (IQ1) was regulated such that the 10-times integral value of the product ion $\mathrm{m} / \mathrm{z} 175.1$ from the precursor ion $\mathrm{m} / \mathrm{z} 906.7$ was $>6 \times 10^{6}$ counts/s (scan rate, 10 Daltons/s; scan range, $\mathrm{m} / z, 172.1$ to 178.1 ). Accordingly, our LC-MS/MS system was operated under the following conditions: $-9.5 \mathrm{~V}$; IQ1, 2800V; CEM. The ESI source was operated as follows: electrode voltage, $5.5 \mathrm{kV}$; source temperature, $700^{\circ} \mathrm{C}$; assisted gas flow, $70 \mathrm{psi}$; nebulizer gas flow, 70 psi; counter gas flow, 30 psi. The MS acquisition parameters for AVP were optimized as follows: de-clustering potential, $80 \mathrm{~V}$; collision energy, $23 \mathrm{~V}$. Data were collected in selected ion monitoring (SIM) mode using a transition of $\mathrm{m} / \mathrm{z}$ 542.8 (doubly protonated ion) $\rightarrow m / z, 328.3$ for AVP. The MS acquisition parameters for IS were optimized as follows: de-clustering potential, $67 \mathrm{~V}$; collision energy, $23.3 \mathrm{eV}$. Data were collected in SIM mode using a transition of $\mathrm{m} / \mathrm{z} 547.9$ (doubly protonated ion) $\rightarrow m / z 328.3$ for the IS.

\section{Method validation}

The method was validated for linearity, accuracy, precision, matrix effects, and recovery. Calibration curves were constructed by comparing peak-area ratios of AVP and those of the IS by weighted $\left(1 / x^{2}\right)$ least-squares linear regression analysis. The precision and accuracy for AVP in human plasma were calculated using four different QC samples (endogenous baseline, endogenous baseline $+0.400 \mathrm{pmol} / \mathrm{L}$ AVP, endogenous baseline $+4.00 \mathrm{pmol} / \mathrm{L} \mathrm{AVP}$, and endogenous baseline + $64.0 \mathrm{pmol} / \mathrm{L}$ AVP). The absolute recovery, extract efficiency, and matrix effect were determined using spiked plasma samples $(n=5)$ with AVP concentrations of 4.00 and $64.0 \mathrm{pmol} / \mathrm{L}$. The absolute recovery was calculated as the peak area of the AVP sample spiked before extraction, divided by the peak area of the neat sample at the same concentration. The extraction efficiency was calculated by dividing the peak area of the AVP sample spiked before extraction by that derived from an equimolar sample of human plasma after extraction. The matrix effect was calculated by dividing the peak area of the AVP sample in plasma spiked after extraction by that of a neat equimolar AVP solution. Method validation was carried out in accordance with a summary report concerning the conference on Bioanalytical Method Validation. ${ }^{21}$

\section{Results and Discussion}

Mass spectra for selecting the ion transition for LC-MS/MS

The ion transition for the quantitation of AVP using selected reaction monitoring (SRM) was determined by the direct infusion of AVP and the IS. AVP and the IS yielded protonated molecules of $\mathrm{m} / \mathrm{z} 1084.4$ and 1094.7, respectively, as revealed by precursor ion scanning using ESI (Figs. 1A and 1C). Doubly charged ions $[\mathrm{M}+2 \mathrm{H}]^{2+}$ of AVP and IS were observed with $\mathrm{m} / \mathrm{z}$ ratios of 542.8 and 547.9, respectively. The product ion mass spectra from doubly charged ions of AVP and IS had an $\mathrm{m} / \mathrm{z}$ of 120.3 as $(\mathrm{F})^{+}$and an $\mathrm{m} / \mathrm{z}$ of 328.3 as $\left(\mathrm{y}_{3}\right)^{+}$(Figs. 1B and 1C). This fragmentation was favorable because both compounds have a disulfide link between the two cysteine residues, and thus $\mathrm{y}$-type fragmentation occurs, yielding $\mathrm{y}_{3}$ ions as the predominant products. $^{22}$

\section{Effect of the mobile-phase solvent on sensitivity}

Figure 2 shows the effect of the aqueous solvent acid concentration of the mobile phase (using two different organic solvent mobile phases) on the assay sensitivity. We found that a low acid concentration in the aqueous mobile phase produced higher sensitivity for AVP, using methanol or acetonitrile as the organic mobile phase. The best sensitivity was achieved with a combination of a $0.01 \%$ aqueous acetic acid solution and methanol. The sensitivity was improved by approximately 3.5 -fold compared to with when it was used a $0.03 \%$ formic 


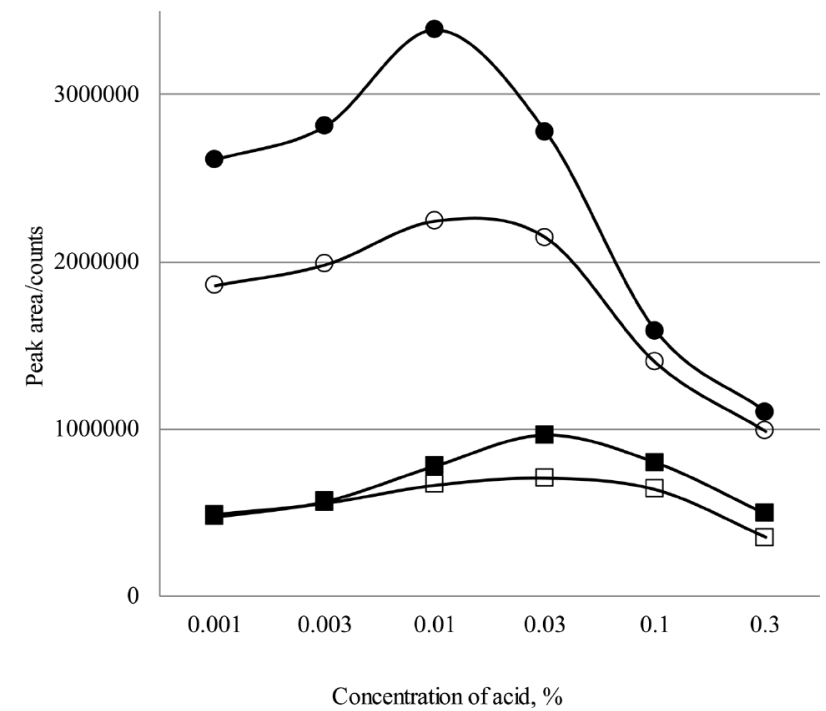

Fig. 2 Ionization effect of AVP as a function of the mobile-phase solvent. ๑, Acetic acid with methanol; $\bigcirc$, formic acid with methanol; $\square$, acetic acid with acetonitrile; $\square$, formic acid with acetonitrile.

acid aqueous solution and acetonitrile. The use of a $0.1 \%$ formic acid aqueous solution and acetonitrile improved the detection sensitivity of peptide quantitation. ${ }^{23-27}$ However, in this work, our comparison revealed that methanol was better than acetonitrile in terms of sensitivity, and that a low acetic acid concentration $(0.01 \%)$ was preferable over the use of formic acid. In general, acetonitrile is used in ESI ionization, because an organic solvent with low viscosity and a low boiling point is preferred for ionization. ${ }^{28}$ However, we observed that methanol promoted ionization to a greater extent than acetonitrile, despite methanol $\left(0.59 \mathrm{mPa} \cdot \mathrm{s}, 20^{\circ} \mathrm{C}\right)$ having a 1.7 -fold higher viscosity than acetonitrile $\left(0.35 \mathrm{mPa} \cdot \mathrm{s}, 20^{\circ} \mathrm{C}\right)$. The charged droplets were generating the constant viscosity required for the ionization of peptides, such as AVP.

Effect of a narrow electrode and the addition of dichloromethane on the assay sensitivity

The generation efficiency of charged droplets from the electrode greatly depends on the flow rate of the mobile phase. If the flow rate is high, it becomes difficult to produce fine droplets since the ionic strength decreases. ${ }^{28}$ We therefore confirmed that the inner diameter difference of the electrode further improved the sensitivity. Figure 3 shows a comparison of the sensitivity observed when using either a narrow electrode (65 $\mu \mathrm{m}$ diameter) or a normal electrode $(100 \mu \mathrm{m}$ diameter $)$, with respect to the AVP detection. Using the $65-\mu \mathrm{m}$ electrode resulted in a nearly 6-fold increase in sensitivity compared to the results obtained with the normal $100-\mu \mathrm{m}$ electrode. The biological matrix can affect the ion suppression and enhancement effects of ESI. ${ }^{29,30}$ The simplest method used to control this matrix effect is to reduce the volume of plasma used for the measurement as much as possible. It has been reported that adding derivatives to the mobile phase can enhance the sensitivity of ESI. ${ }^{31,32}$ Therefore, we studied the effects of including dichloromethane on the sensitivity in a mobile phase, using the narrow electrode. Moreover, derivatives were added to the mobile phase, in an attempt to improve the sensitivity by increasing the elution strength. Acetone and dichloromethane are known additives for enhancing the dissolution power. The addition of acetone reduced the sensitivity (data not shown).

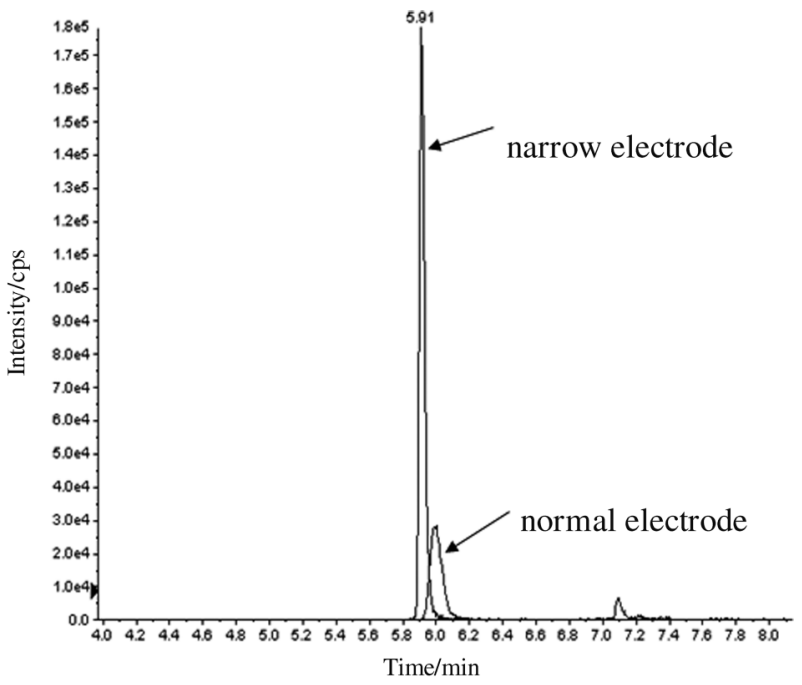

Fig. 3 Sensitivities of measurements obtained using a narrow electrode $(65 \mu \mathrm{m}$ diameter $)$ compared with those obtained using a normal electrode (100 $\mu \mathrm{m}$ diameter) for selected reactions. Selected reaction monitoring was performed with a $200 \mathrm{ng} / \mathrm{mL}$ AVP sample, with the product ion being $\mathrm{m} / \mathrm{z}, 328.3$ and the parent ion being $\mathrm{m} / \mathrm{z}$ 542.8 .

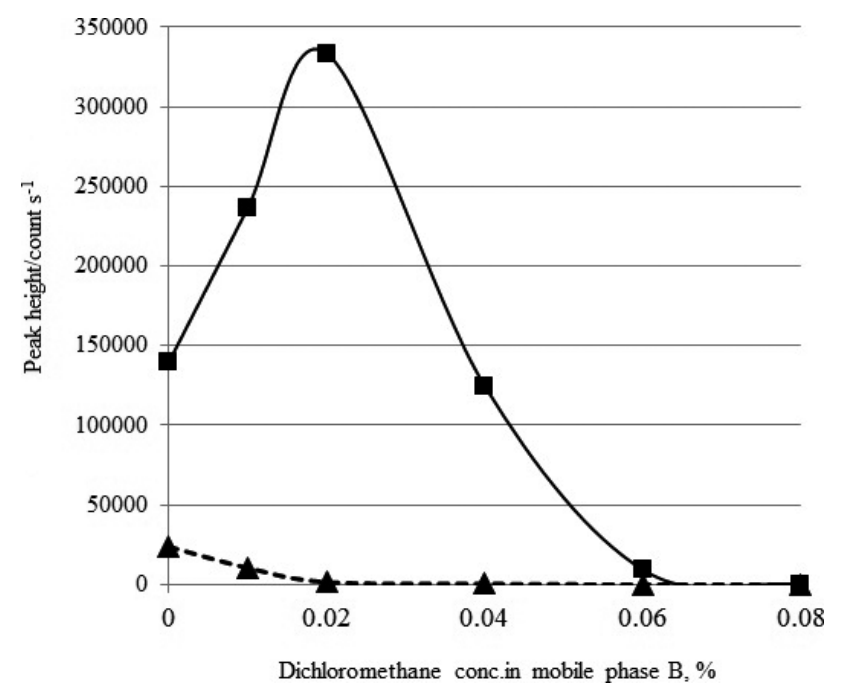

Fig. 4 Effects of the mobile-phase additive, dichloromethane, on the sensitivity of AVP detection. Closed squares indicate values obtained using a narrow electrode, and closed triangles indicate values obtained using a normal electrode.

However, the addition of dichloromethane, using the narrow electrode, tended to boost the AVP sensitivity. Furthermore, the concentration of dichloromethane at the time of addition was approximately the same as that of the acids optimal concentration. In the mobile phase, at a concentration of $0.02 \%$, dichloromethane improved sensitivity by approximately two-fold (Fig. 4). However, the sensitivity tended to decrease with a further increase in the dichloromethane concentration. Moreover, by using the normal electrode, the sensitivity was significantly reduced by the addition of dichloromethane, presumably resulting from the size of the charged droplets to be generated. Dichloromethane was effective in the process of ion evaporation, after the formation of charged droplets, and the proton donor was found to be impaired by any excess addition of the analyte. 


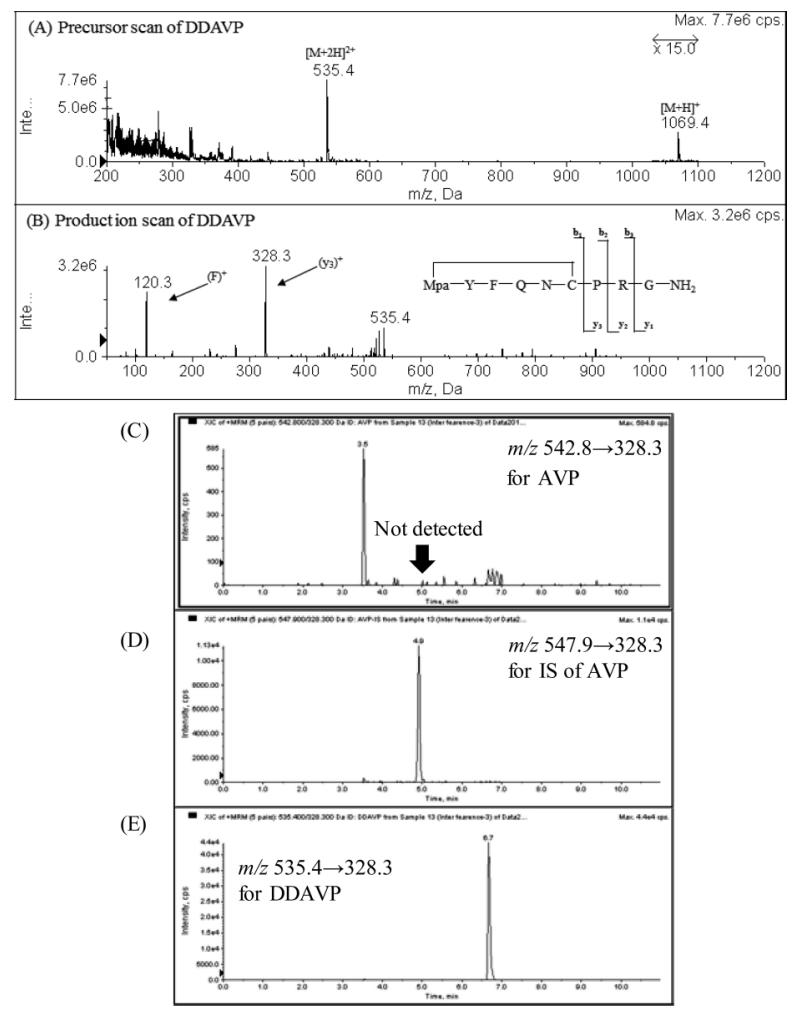

Fig. 5 ESI-positive mass spectra of DDAVP. Precursor ion mass spectra of DDAVP (A). Product ion mass spectra of DDAVP (B). Extracted ion chromatograms for AVP from control serum (0 pmol/L AVP) (C), IS of AVP (D) and DDAVP (E).

\section{Confirmation of the interference from DDAVP}

The amino acid sequence of DDVAP is Mpr-Tyr-Phe-GlnAsn-Cys-Pro-D-Arg-Gly- $\mathrm{NH}_{2}$, with a monoisotopic mass of 1068.43. The ion transition of DDAVP was verified in a manner similar to that of AVP. DDAVP yielded protonated molecules at an $\mathrm{m} / \mathrm{z}$ ratio of 1069.4 , as shown by precursor ion scanning using ESI (Fig. 5A). Doubly charged $[\mathrm{M}+2 \mathrm{H}]^{2+}$ ions of DDAVP were observed at $\mathrm{m} / \mathrm{z}$ ratios of 535.4. Product ion mass spectra from doubly charged ions of DDAVP had an $\mathrm{m} / \mathrm{z}$ of 120.3 as

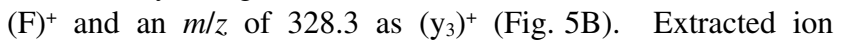
chromatograms for AVP from control serum ( $0 \mathrm{pmol} / \mathrm{L}$ AVP) are shown in Fig. 5C $(\mathrm{m} / \mathrm{z}, 542.8 \rightarrow 328.3)$, the IS of AVP is shown in Fig. 5D $(\mathrm{m} / \mathrm{z} 547.9 \rightarrow 328.3)$ and DDAVP is shown in Fig. 5E $(\mathrm{m} / \mathrm{z}, 535.4 \rightarrow 328.3)$. These ion transitions of AVP, IS of AVP and DDAVP did not overlap on the LC-MS/MS method.

\section{Extracted ion chromatograph}

Extracted ion chromatograms for AVP $(\mathrm{m} / z 542.8 \rightarrow 328.3)$ from control serum and human plasma are shown in Fig. 6 . Figure 6A shows that the IS for AVP and DDAVP did not contribute to any interference peaks that would affect the ability of our experimental setup to quantitate AVP. An LLOQ of AVP $(0.200 \mathrm{pmol} / \mathrm{L})$ was injected into the LC-MS/MS; this yielded $15.2 \mathrm{fg}$ on the column with a signal-to-noise ratio of 9 (Fig. 6B). Figure 6C shows an extracted ion chromatogram of a low-QC $(0.400 \mathrm{pmol} / \mathrm{L}$ of AVP) sample, which included the endogenous baseline. The total quantitative value of AVP detected was $3.59 \mathrm{pmol} / \mathrm{L}$. This result was consistent with the endogenous baseline quantitative value $(3.16 \mathrm{pmol} / \mathrm{L})$ that was obtained when the QC sample concentration $(0.400 \mathrm{pmol} / \mathrm{L})$ was subtracted from the total quantitative value $(3.59 \mathrm{pmol} / \mathrm{L})$.
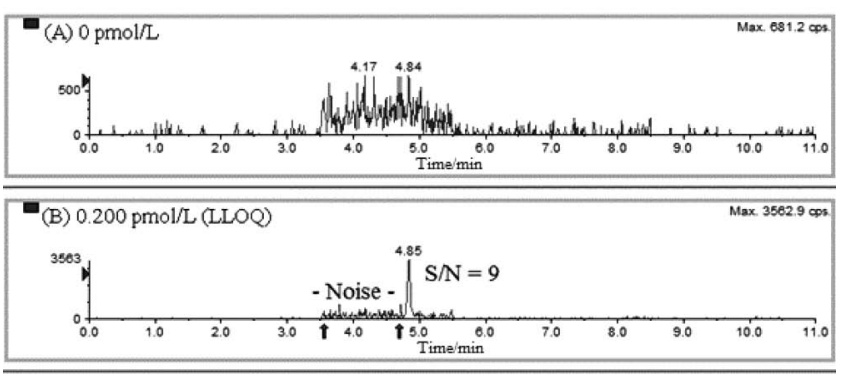

(C) Low QC (3.59 pmol/L: $0.400 \mathrm{pmol} / \mathrm{L}+3.16 \mathrm{pmol} / \mathrm{L}$ endogenous beseline) Max. $5.704 \mathrm{cos}^{-10}$

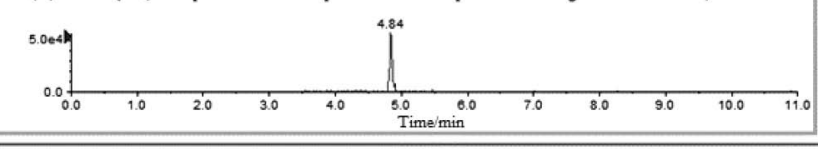

- (D) Human plasma sample No.21 (0.209 pmol/L)

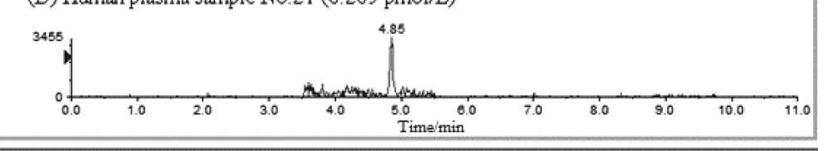

- (E) Human plasma sample No.6 $(0.987 \mathrm{pmol} / \mathrm{L})$

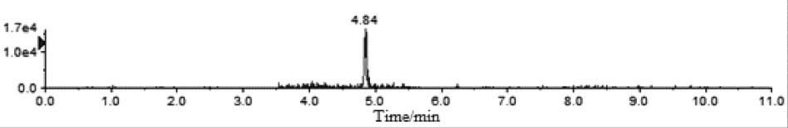

(F) Human plasma sample No.1 (3.28 pmol/L) Max. 5.264 cos

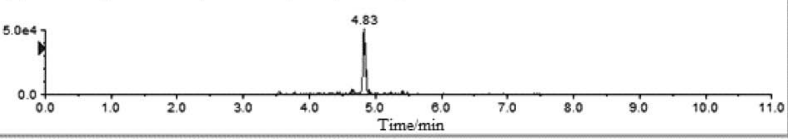

Fig. 6 Selected reaction monitoring of extracted ion chromatograms for AVP using control human serum and plasma with the product ion being $\mathrm{m} / \mathrm{z}, 328.3$ and the parent ion being $\mathrm{m} / \mathrm{z}$ 542.8. (A) $0 \mathrm{pmol} / \mathrm{L}$; (B) $0.200 \mathrm{pmol} / \mathrm{L}$ AVP spiked into control serum; (C) low QC sample (0.400 pmol/L AVP-spiked commercial human plasma); (D - F) human plasma samples from volunteers.

Table 1 Calibration curves of intraday measurements $(N=5)$ of AVP in control serum by LC-MS/MS analysis

\begin{tabular}{cccc}
\hline $\begin{array}{c}\text { Expected } \\
\text { concentration/ } \\
\text { pmol L }\end{array}$ & $\begin{array}{c}\text { Observed } \\
\text { concentration/ } \\
\text { pmol L }\end{array}$ & $\begin{array}{c}\text { RSD, } \\
\text { mean } \pm \text { SD }^{\mathrm{a}}\end{array}$ & $\begin{array}{c}\text { RE, } \\
\%\end{array}$ \\
\hline 0.200 & $0.202 \pm 0.012$ & 5.9 & 1.1 \\
0.400 & $0.397 \pm 0.026$ & 6.6 & -0.7 \\
2.00 & $1.97 \pm 0.11$ & 5.6 & -1.4 \\
4.00 & $3.98 \pm 0.11$ & 2.7 & -0.5 \\
20.0 & $20.1 \pm 0.6$ & 3.0 & 0.7 \\
40.0 & $40.3 \pm 1.9$ & 4.7 & 0.7 \\
80.0 & $80.3 \pm 4.3$ & 5.3 & 0.3 \\
$\gamma($ mean \pm SD) & $0.9992 \pm 0.0002$ & - & - \\
Slope (mean \pm SD) & $0.0449 \pm 0.0009$ & - & - \\
\hline
\end{tabular}

a. The values shown are the mean $\pm \mathrm{SD}$.

Figures $6 \mathrm{D}$ to $6 \mathrm{~F}$ show ion chromatographs derived from analyses of plasma from volunteers. The LLOQ value of volunteer sample No. 21 was close to $0.200 \mathrm{pmol} / \mathrm{L}$, and there was no interference from impurities that affected the quantitation. Combined with the excellent LLOQ results in the calibration sample prepared from control serum (Fig. 6B), these data indicate that the method has greater selectivity for the 
Table 2 Interday and intraday precision (\%RSD) and accuracy (\%RE) of the LC-MS/MS assay used to quantitate AVP in human pooled plasma

\begin{tabular}{|c|c|c|c|c|c|c|c|c|}
\hline \multirow[b]{2}{*}{ Expected concentration } & \multicolumn{4}{|c|}{ Interday } & \multicolumn{4}{|c|}{ Intraday } \\
\hline & $\begin{array}{c}\text { Observed } \\
\text { concentration/ } \\
\text { pmol L-1 a }\end{array}$ & $\begin{array}{l}\mathrm{RSD}, \\
\%\end{array}$ & RE, \% & $\begin{array}{l}\text { Subtracted } \\
\text { concentration/ } \\
\text { pmol L-1 b }\end{array}$ & $\begin{array}{c}\text { Observed } \\
\text { concentration/ } \\
\text { pmol L-1 a }\end{array}$ & $\begin{array}{l}\text { RSD, } \\
\%\end{array}$ & $\begin{array}{c}\mathrm{RE} \\
\%\end{array}$ & $\begin{array}{c}\text { Subtracted } \\
\text { concentration/ } \\
\text { pmol L } \text { L }^{-1 \mathrm{~b}}\end{array}$ \\
\hline Endogenus baseline & $3.13 \pm 0.03$ & 0.9 & Not calculated & Not calculated & $3.13 \pm 0.08$ & 2.4 & Not calculated & Not calculated \\
\hline $\begin{array}{l}\text { Low QC }(0.400 \mathrm{pmol} / \mathrm{L}+ \\
\text { endogenus baseline })\end{array}$ & $3.53 \pm 0.07$ & 1.9 & 0.2 & 0.406 & $3.56 \pm 0.05$ & 1.4 & 0.8 & 0.428 \\
\hline $\begin{array}{l}\text { Middle QC ( } 4.00 \mathrm{pmol} / \mathrm{L}+ \\
\text { endogenus baseline) }\end{array}$ & $7.17 \pm 0.10$ & 1.3 & 0.6 & 4.04 & $7.27 \pm 0.22$ & 3.0 & 2.0 & 4.14 \\
\hline $\begin{array}{l}\mathrm{High} \text { QC ( } 64.0 \mathrm{pmol} / \mathrm{L}+ \\
\text { endogenus baseline) }\end{array}$ & $66.9 \pm 1.61$ & 2.4 & -0.3 & 63.8 & $67.0 \pm 0.8$ & 1.2 & -0.2 & 63.9 \\
\hline
\end{tabular}

a. The values shown are the mean \pm SD. b. The subtracted concentration was calculated as the mean observed concentration for each QC sample minus the mean observed concentration of the endogenous baseline.

Table 3 Absolute recovery, extraction efficiency, and matrix effect of AVP in human pooled plasma

\begin{tabular}{cccc}
\hline $\begin{array}{c}\text { Concentration/ } \\
\text { pmol L }^{-1}\end{array}$ & $\begin{array}{c}\text { Absolute } \\
\text { recovery, } \%^{\mathrm{a}}\end{array}$ & $\begin{array}{c}\text { Extract efficiency, } \\
\%^{\mathrm{a}}\end{array}$ & $\begin{array}{c}\text { Matrix } \\
\text { effect, \% }\end{array}$ \\
\hline 4.00 & $71.5 \pm 8.1$ & $70.2 \pm 7.2$ & 101.9 \\
64.0 & $74.1 \pm 6.9$ & $73.3 \pm 6.2$ & 101.1 \\
\hline
\end{tabular}

a. The values shown are the mean \pm SD.

quantitation of AVP in human plasma, compared to previous approaches.

\section{Assay validation results}

Titration of AVP from 0.200 to $80.0 \mathrm{pmol} / \mathrm{L}(N=5$, Table 1$)$ yielded a good linear response. The coefficient of correlation $(\gamma) \pm$ SD and slope of the calibration curves were $0.9992 \pm$ 0.0002 and $0.0499 \pm 0.0009$, respectively. The percent relative error (\%RE) between back-calculated standard concentrations and nominal values typically ranged from -1.4 to $1.1 \%$. The interday precision values ranged from 1.3 to $2.4 \%$, while the interday accuracy values ranged from -0.3 to $0.6 \%$. The intraday precision values ranged from 1.2 to $3.0 \%$, and the intraday accuracy values ranged from -0.2 to $2.0 \%$ for AVP $(N=5$, Table 2). Excellent agreement and very little variation between the interday and intraday measurements were observed after normalization to the endogenous AVP concentration in pooled human plasma (Table 2). The absolute recoveries of AVP (4.00 and $64.0 \mathrm{pmol} / \mathrm{L}, N=5)$ were $71.5 \pm 8.1$ to $74.1 \pm$ $6.9 \%$. The SPE efficiency ranged from $70.2 \pm 7.2$ to $73.3 \pm$ $6.2 \%$ for AVP (Table 3). Although these recoveries were approximately $70 \%$, the change in recovery by the matrix ranged from 101.9 to $101.1 \%$ (= calculated matrix effect values were fairly low $(1.1-1.9 \%))$. Although the efficiency for extracting AVP from the human plasma was inferior, contaminants in the organism did not suppress or promote ionization. It was shown to be a method that can provide stable measurements. All of the validation data, such as accuracy, precision, and inter-day repeatability, were within the required limits.

\section{Application}

The diagnostics section in the hospital used an RIA antibody kit (AVP-RIA kit, 0.14 pmol/L as LLOQ; LSI Medience Corp.;
Table 4 AVP concentrations measured in plasma samples from human volunteers by the RIA and LC-MS/MS methods

\begin{tabular}{cccc}
\hline \multirow{2}{*}{$\begin{array}{c}\text { Sample } \\
\text { No. }\end{array}$} & \multicolumn{2}{c}{ AVP concentration/pmol L } & \multicolumn{1}{c}{$\begin{array}{c}\text { \% of } \\
\text { difference }\end{array}$} \\
\cline { 2 - 3 } & RIA & LC-MS/MS & \\
\hline 1 & 3.52 & 3.28 & 7.1 \\
2 & 7.52 & 6.99 & 7.3 \\
3 & 10.1 & 11.2 & -10.3 \\
4 & 0.797 & 0.741 & 7.3 \\
5 & 0.399 & 0.414 & -3.7 \\
6 & 1.11 & 0.987 & 11.7 \\
7 & 0.335 & 0.301 & 10.7 \\
8 & 0.705 & 0.781 & -10.2 \\
9 & 0.89 & 0.778 & 13.4 \\
10 & 0.246 & 0.235 & 4.6 \\
11 & 0.33 & 0.289 & 13.2 \\
12 & 0.457 & 0.511 & -11.2 \\
13 & 6.02 & 6.38 & -5.8 \\
14 & 4.72 & 4.28 & 9.8 \\
15 & 7.21 & 7.62 & -5.5 \\
16 & 7.74 & 7.08 & 8.9 \\
17 & 0.362 & 0.405 & -11.2 \\
18 & 0.511 & 0.484 & 5.4 \\
19 & 0.587 & 0.493 & 17.4 \\
20 & 1.47 & 1.23 & 17.8 \\
21 & 0.188 & 0.209 & -10.6 \\
22 & 1.38 & 1.24 & 10.7 \\
\hline
\end{tabular}

a. \% Difference

$=$ (RIA quantitative value) - (LC-MS/MS quantitative value)

$=\frac{\text { Mean concentration determined by RIA and LC-MS/MS }}{\text { L }} \times 100$.

Tokyo, Japan) to measure the AVP concentrations in 22 human plasma samples from volunteers. We compared these results to those obtained using our new method (Table 4). Percentage differences were calculated as $100 \times$ (RIA quantitative value LC-MS/MS quantitative value)/(mean concentration determined by RIA and LC/MS-MS). Moreover, both methods were able to quantify low AVP levels $(<1 \mathrm{pmol} / \mathrm{L})$ in 12 out of 22 plasma samples. These findings indicate that the LC-MS/MS approach for measurements of AVP in human plasma samples produced both sensitive and selective (i.e., interference-free) results. 


\section{Conclusions}

Here, we describe the measurement of AVP in human plasma using an LC-MS/MS instrument equipped with a narrow electrode. This method has excellent sensitivity and selectivity, the latter of which can be enhanced by adding $0.02 \%$ dichloromethane to the mobile phase. Our approach requires extremely small sample amounts, as since reliable results were obtained with only $100 \mu \mathrm{L}$ of human plasma. The method is more quantitative (from $0.200 \mathrm{pmol} / \mathrm{L}$ as the LLOQ to $80.0 \mathrm{pmol} / \mathrm{L}$ as the upper LOQ in human plasma) than the conventional RIA approach. It is highly accurate and precise, and can be used to detect sub-picomolar concentrations of AVP. Our results also indicate that the use of LC-MS/MS equipped with a narrow electrode may overcome some of the technical hurdles associated with the quantification of clinically and biologically significant peptides.

\section{Acknowledgements}

We thank Prof. Yutaka Oiso (Nagoya University Graduate School of Medicine) for helpful discussions regarding the application data.

\section{References}

1. H. K. Caldwell and W. S. Young, III, "Handbook of Neurochemistry and Molecular Neurobiology", ed. R. Lim and A. Lajtha, 2006, Springer, New York, 573.

2. S. M. Babar, Ann. Pharmacother., 2013, 47, 1359.

3. M. Birnbaumer, S. Gilbert, and W. Rosenthal, Mol. Endocrinol., 1994, 8, 886.

4. H. Jinnouchi, E. Araki, N. Miyamura, H. Kishikawa, R. Yoshimura, S. Isami, K. Yamaguchi, H. Iwamatsu, and M. Shichiri, Eur. J. Endocrinol., 1996, 134, 689.

5. A. Jovanović, L. Grbović, I. Žikić, and I. Tulic, Br. J. Pharmacol., 1995, 115, 1295.

6. T. Zenteno-Savin, I. Sada-Ovalle, G. Ceballos, and R. Rubio, Eur. J. Pharmacol., 2000, 410, 15.

7. P. H. Baylis, Int. J. Biochem. Cell Biol., 2003, 35, 1495.

8. D. R. Cool and D. DeBrosse, J. Chromatogr. B: Anal. Technol. Biomed. Life Sci., 2003, 792, 375.

9. G. G. Klee, Clin. Lab. Med., 2004, 24, 1.

10. S. Lundin, P. Melin, and H. Vilhardt, Experientia, 1985, 41, 933.

11. W. A. Sadler, C. P. Lynskey, and K. P. Dawson, Anal.
Biochem., 1984, 143, 272.

12. M. Thevis and W. Schänzer, Analyst, 2007, 132, 287.

13. M. Rauh, J. Chromatogr. B: Anal. Technol. Biomed. Life Sci., 2012, 883-884, 59.

14. C. Hao, J. L. Campbell, U. H. Verkerk, J. C. Le Blanc, and K. W. Siu, J. Am. Soc. Mass Spectrom., 2011, 22, 67.

15. O. S. Mabrouk and R. T. Kennedy, J. Neurosci. Methods, 2012, 209, 127.

16. D. Zhang, D. R. Rios, V. H. Tam, and D. S. Chow, J. Pharm. Biomed. Anal., 2014, 99, 67.

17. R. W. Schrier, "Renal and Electrolyte Disorders", 2010, Lippincott Williams and Wilkins, Philadelphia.

18. R. W. Schrier, "Diseases of the Kidney \& Urinary Tract, 3-Volume Set", 8th ed., 2007, Lippincott Williams and Wilkins, Philadelphia.

19. Y. Tsukazaki and N. Senda, Japan Patent 2014, 238338.

20. C. Doneanu and P. Rainville, Ultrasensitive Quantification Assay for Oxytocin in Human Plasma Using a LC/MS Microfluidics Platform. The 61st American Society for Mass Spectrometry (ASMS) Conference, Minneapolis, MN, 2013, Poster MP532.

21. "Guidance for Industry. Bioanalytical Method Validation", 2001, US Food and Drug Administration.

22. S. Esposito, K. Deventer, G. T'Sjoen, A. Vantilborgh, F. T. Delbeke, A. S. Goessaert, K. Everaert, and P. Van Eenoo, Anal. Bioanal. Chem., 2012, 402, 2789.

23. G. Ali, Y. Wu, S. Nag, and T. Hussein, Anal. Methods, 2014, 6, 215.

24. Z. Chen, M. P. Caulfield, M. J. McPhaul, R. E. Reitz, S. W. Taylor, and N. J. Clarke, Clin. Chem., 2013, 59, 1349.

25. L. Cui, K. Nithipatikom, and W. B. Campbell, Anal. Biochem., 2007, 369, 27.

26. S. Esposito, K. Deventer, G. T'Sjoen, A. Vantilborgh, and P. Van Eenoo, Biomed. Chromatogr., 2013, 27, 240.

27. G. Zhang, Y. Zhang, D. M. Fast, Z. Lin, and R. Steenwyk, Anal. Biochem., 2011, 416, 45.

28. Manual for Development of Chemical Analysis Method Using LC/MS, Ministry of the Environment, Comprehensive Environmental Policy Bureau, Environmental Health Department, Environmental Safety Division, http://www. env.go.jp/chemi/anzen/lcms/.

29. L. L. Jessome and D. A. Volmer, LCGC North Am., 2006, 24, 498

30. D. Remane, M. R. Meyer, D. K. Wissenbach, and H. H. Maurer, Rapid Commun. Mass Spectrom., 2010, 24, 3103.

31. S. Gao, Z. P. Zhang, and H. T. Karnes, J. Chromatogr. B: Anal. Technol. Biomed. Life Sci., 2005, 825, 98.

32. W. Li, J. Zhang, and F. L. S. Tse, "Handbook of LC-MS Bioanalysis", 2013, John Wiley \& Sons, Hoboken. 